\title{
The law of large numbers and a functional equation
}

\author{
by JoAnna Ger and Maciej SAblik (Katowice)
}

Abstract. We deal with the linear functional equation

$$
g(x)=\sum_{i=1}^{r} p_{i} g\left(c_{i} x\right)
$$

where $g:(0, \infty) \rightarrow(0, \infty)$ is unknown, $\left(p_{1}, \ldots, p_{r}\right)$ is a probability distribution, and $c_{i}$ 's are positive numbers. The equation (or some equivalent forms) was considered earlier under different assumptions (cf. [1], [2], [4], [5] and [6]). Using Bernoulli's Law of Large Numbers we prove that $g$ has to be constant provided it has a limit at one end of the domain and is bounded at the other end.

1. Introduction. In [4] the authors asked for the conditions under which any solution of the equation

$$
f(x-\varphi(x))+f(x+\varphi(x))=2 f(x)
$$

is affine. The equation $(\mathrm{J})$ is called the Jensen equation on the graph of the function $\varphi$. For $\varphi$ linear, say $\varphi(x)=\alpha x, x \in(0, \infty),(\mathrm{J})$ leads to the equation

$$
g(x)=\frac{c}{2} g(c x)+\frac{d}{2} g(d x)
$$

where $g(x)=f(x) / x, c=1+\alpha$ and $d=1-\alpha$. Obviously, $(*)$ is a particular case of (E) which is our main point of interest. Thus one motivation for the present paper is to extend our previous results.

Another motivation comes from [1] and [6] (cf. also [5]), where the following equation has been considered:

$$
G(t)=\sum_{i=1}^{r} A_{i} G\left(t+a_{i}\right)
$$

where $G: \mathbb{R} \rightarrow \mathbb{R}$ is unknown, $A_{i}$ 's are positive, and $a_{i}$ 's are different from 0 .

1991 Mathematics Subject Classification: 39B22, 39A10.

Key words and phrases: functional equation, law of large numbers, Jensen equation on curves, bounded solutions, difference equation. 
Suppose that $\lambda \in \mathbb{R}$ is a solution of the characteristic equation of (L), i.e.

$$
\sum_{i=1}^{r} A_{i} e^{\lambda a_{i}}=1
$$

Then it easy to check that $G$ solves (L) if and only if $g:(0, \infty) \rightarrow \mathbb{R}$ given by

$$
g(x)=x^{-\lambda} G(\ln x)
$$

solves (E) with $p_{i}=A_{i} e^{\lambda a_{i}}$ and $c_{i}=e^{a_{i}}, i \in\{1, \ldots, r\}$. The equation (L) has been studied by the aforementioned authors either under the assumption that solutions are continuous and bounded (G. Derfel, who moreover uses probability methods to prove the results) or measurable and nonnegative (M. Laczkovich) or satisfy some asymptotic conditions (J. Baker). It might be interesting that M. Pycia in [7] when dealing in particular with (L) with equality replaced by inequality, assumes measurability and asymptotic conditions. In the present paper we also impose some asymptotic conditions, which is another consequence of our original interest in solving the Jensen equation on curves. In [4], following several authors dealing with a similar problem for the Cauchy equation on curves, we looked for solutions of $(\mathrm{J})$ which are differentiable at 0 and such that the quotient $f(x) / x$ is bounded at infinity. Our present results concerning (E) are of a similar type.

2. Preliminaries. Let $(\Omega, \mathcal{A}, \mathcal{P})$ be a probability space, let $r \in \mathbb{N}$ be a positive integer and fix $p_{1}, \ldots, p_{r} \geq 0$ such that $\sum_{i=1}^{r} p_{i}=1$. We consider a sequence $\left(X_{n}\right)_{n \in \mathbb{N}}$ of vector random variables, $X_{n}=\left(X_{n, 1}, \ldots, X_{n, r}\right)$, where $X_{n, i}: \Omega \rightarrow \mathbb{R}$ for $i \in\{1, \ldots, r\}$, and assume that for every $n \in \mathbb{N}$ the random variable $X_{n}$ has polynomial distribution, i.e. for every $k_{1}, \ldots, k_{r} \in$ $\{0,1, \ldots, n\}$ such that $k_{1}+\ldots+k_{r}=n$,

$$
\mathcal{P}\left(\left\{\omega \in \Omega: X_{n, 1}(\omega)=k_{1}, \ldots, X_{n, r}(\omega)=k_{r}\right\}\right)=\frac{n !}{k_{1} ! \ldots k_{r} !} p_{1}^{k_{1}} \ldots p_{r}^{k_{r}} .
$$

We start with the following

Lemma 2.1. If $\left(X_{n}\right)_{n \in \mathbb{N}}, X_{n}=\left(X_{n, 1}, \ldots, X_{n, r}\right), n \in \mathbb{N}$, is a sequence of vector random variables with polynomial distribution, then for every $\delta>0$,

$$
\lim _{n \rightarrow \infty} \mathcal{P}\left(\left\{\omega \in \Omega: \max _{1 \leq i \leq r}\left|\frac{X_{n, i}(\omega)}{n}-p_{i}\right|<\delta\right\}\right)=1 .
$$

Proof. Fix $\delta>0$. For $i \in\{1, \ldots, r\}$ set

$$
A_{i}^{n}=\left\{\omega \in \Omega:\left|\frac{X_{n, i}(\omega)}{n}-p_{i}\right|<\delta, \sum_{j=1, j \neq i}^{r} X_{n, j}(\omega)=n-X_{n, i}(\omega)\right\} .
$$


Then

$$
\bigcap_{i=1}^{n} A_{i}^{n}=\left\{\omega \in \Omega: \max _{1 \leq i \leq r}\left|\frac{X_{n, i}(\omega)}{n}-p_{i}\right|<\delta\right\} .
$$

It is obvious that if we prove

$$
\lim _{n \rightarrow \infty} \mathcal{P}\left(A_{i}^{n}\right)=1 \quad \text { for } i \in\{1, \ldots, r\},
$$

then we get (1). By symmetry it is enough to show that (2) holds for $i=r$. We have

$$
\begin{aligned}
\mathcal{P}\left(A_{r}^{n}\right)= & \sum_{\left\{k_{r}:\left|k_{r} / n-p_{r}\right|<\delta\right\}} \sum_{k_{1}+\ldots+k_{r-1}=n-k_{r}} \frac{n !}{k_{1} ! \ldots k_{r} !} p_{1}^{k_{1}} \ldots p_{r-1}^{k_{r-1}} p_{r}^{k_{r}} \\
= & \sum_{\left\{k_{r}:\left|k_{r} / n-p_{r}\right|<\delta\right\}} \frac{n !}{k_{r} !\left(n-k_{r}\right) !} p_{r}^{k_{r}} \\
& \times \sum_{k_{1}+\ldots+k_{r-1}=n-k_{r}} \frac{\left(n-k_{r}\right) !}{k_{1} ! \ldots k_{r-1} !} p_{1}^{k_{1}} \ldots p_{r-1}^{k_{r-1}} \\
= & \sum_{\left\{k_{r}:\left|k_{r} / n-p_{r}\right|<\delta\right\}} \frac{n !}{k_{r} !\left(n-k_{r}\right) !} p_{r}^{k_{r}}\left(1-p_{r}\right)^{n-k_{r}} \\
= & \mathcal{P}\left(\left\{\omega \in \Omega:\left|\frac{Y_{n, r}(\omega)}{n}-p_{r}\right|<\delta\right\}\right),
\end{aligned}
$$

where $Y_{n, r}: \Omega \rightarrow \mathbb{R}, n \in \mathbb{N}$, is a random variable with Bernoulli distribution

$$
\mathcal{P}\left(Y_{n, r}=k_{r}\right)=\left(\begin{array}{c}
n \\
k_{r}
\end{array}\right) p_{r}^{k_{r}}\left(1-p_{r}\right)^{1-k_{r}} .
$$

Using Bernoulli's law of large numbers (cf. [3], Chapter VI, §4), we get (2).

In the sequel we will deal with the equation

$$
g(x)=\sum_{i=1}^{r} p_{i} g\left(c_{i} x\right)
$$

assuming that

(H) $\quad p_{i}>0, c_{i} \neq 1,1 \leq i \leq r, 0<c_{1}<\ldots<c_{r}, \sum_{i=1}^{r} p_{i}=1$, and $\prod_{i=1}^{r} c_{i}^{p_{i}} \neq 1$.

Consider the characteristic equation for (E), i.e.

$$
\sum_{i=1}^{r} p_{i} c_{i}^{\lambda}=1
$$

Denote by $\Lambda$ the set of roots of (3). In view of $(\mathrm{H})$ we have $0 \in \Lambda$. Using simple calculus methods to the function $\mathbb{R} \ni \lambda \rightarrow \sum_{i=1}^{r} p_{i} c_{i}^{\lambda}-1 \in \mathbb{R}$ one can show that card $\Lambda \leq 2$ and the following holds. 
Lemma 2.2. Assume that $(\mathrm{H})$ holds. We have

(i) if $c_{1}>1$ or $c_{r}<1$ then $\Lambda=\{0\}$;

(ii) if $c_{1}<1<c_{r}$ then $\Lambda=\{0, \lambda\}$; moreover,

while

$$
\prod_{i=1}^{r} c_{i}^{p_{i}}>1 \Rightarrow \lambda<0 \text { and } \prod_{i=1}^{r} c_{i}^{p_{i} c_{i}^{\lambda}}<1
$$

$$
\prod_{i=1}^{r} c_{i}^{p_{i}}<1 \Rightarrow \lambda>0 \text { and } \prod_{i=1}^{r} c_{i}^{p_{i} c_{i}^{\lambda}}>1
$$

3. Main results. Let us prove first the following extension of Theorem 1 of $[4]$.

Proposition 3.1. Let $g:(0, \infty) \rightarrow \mathbb{R}$ be a solution of equation (E). If either
$\left(\mathrm{A}_{1}\right)$
$g$ is bounded in the vicinity of 0 ,
$\left(\mathrm{A}_{2}\right)$
$\lim _{x \rightarrow \infty} g(x)=a \in[-\infty, \infty]$, and
$\left(\mathrm{A}_{3}\right)$$$
\prod_{i=1}^{r} c_{i}^{p_{i}}>1
$$
or
$\left(\mathrm{B}_{1}\right) \quad g$ is bounded in the vicinity of $\infty$,
$\left(\mathrm{B}_{2}\right) \quad \lim _{x \rightarrow 0^{+}} g(x)=a \in[-\infty, \infty]$, and
$\left(\mathrm{B}_{3}\right) \quad \prod_{i=1}^{r} c_{i}^{p_{i}}<1$,

then $a \in \mathbb{R}$ and $g(x)=a, x \in(0, \infty)$.

Pr o of. Assume that $\left(\mathrm{A}_{1}\right)-\left(\mathrm{A}_{3}\right)$ hold. We first show that for every $R>0$ there exists a $B_{R}>0$ such that

$$
|g(x)| \leq B_{R}
$$

for every $x \in(0, R)$.

It follows from $\left(\mathrm{A}_{3}\right)$ that $c_{r}=\max \left\{c_{1}, \ldots, c_{r-1}\right\}>1$ and hence

$$
\alpha:=\min \left\{1,1 / c_{1}, \ldots, 1 / c_{r-1}\right\} \cdot c_{r}>1 .
$$

Let $d_{0}>0$ and $\beta_{0}>0$ be such that for every $x \in\left(0, d_{0}\right)$,

$$
|g(x)| \leq \beta_{0} .
$$

Fix $R>0$ and let $x \in\left(0, d_{1}\right)$ where $d_{1}:=\alpha d_{0}$. Then $x / c_{r}<d_{0}$ and 
$\left(c_{i} / c_{r}\right) x<d_{0}, i \in\{1, \ldots r-1\}$. From (E) and (5) we get

$$
\begin{aligned}
|g(x)| & =\left|\frac{1}{p_{r}}\left[g\left(\frac{x}{c_{r}}\right)-\sum_{i=1}^{r-1} p_{i} g\left(\frac{c_{i} x}{c_{r}}\right)\right]\right| \\
& \leq \frac{1}{p_{r}}\left(\left|g\left(\frac{x}{c_{r}}\right)\right|+\sum_{i=1}^{r-1} p_{i}\left|g\left(\frac{c_{i} x}{c_{r}}\right)\right|\right) \\
& \leq \frac{\beta_{0}}{p_{r}}\left(1+\sum_{i=1}^{r-1} p_{i}\right)=\frac{\beta_{0}}{p_{r}}\left(2-p_{r}\right)=\beta_{0}\left(\frac{2}{p_{r}}-1\right)=: \beta_{1} .
\end{aligned}
$$

Using the same argument, we prove that for every $n \in \mathbb{N}$ there exists a $\beta_{n}>0$ such that

$$
|g(x)| \leq \beta_{n} \leq\left(\frac{2}{p_{r}}-1\right)^{n} \beta_{0}
$$

for every $x \in\left(0, d_{n}\right)$ where $d_{n}:=\alpha^{n} d_{0}$. Since $\alpha>1$ we have $\alpha^{N} d_{0}>R$ for some $N \in \mathbb{N}$. In view of (6) it is enough to put $B_{R}:=\beta_{N}$.

For every $n \in \mathbb{N}$, put

$$
\Delta_{n}:=\left\{\left(k_{1}, \ldots, k_{r}\right) \in(\mathbb{N} \cup\{0\})^{r}: k_{1}+\ldots+k_{r}=n\right\} .
$$

An easy induction shows that (E) implies

(7) $g(x)=\sum_{\left(k_{1}, \ldots, k_{r}\right) \in \Delta_{n}} \frac{n !}{k_{1} ! \ldots k_{r} !} p_{1}^{k_{1}} \ldots p_{r}^{k_{r}} g\left(c_{1}^{k_{1}} \ldots c_{r}^{k_{r}} x\right), \quad x \in(0, \infty)$,

for $n \in \mathbb{N}$. First, we will prove that $a \in \mathbb{R}$. Indeed, suppose that $a=\infty$. Fix $D>0$ and let $R>0$ be such that

$$
g(x) \geq D
$$

for every $x \geq R$. Let $\varepsilon>0$ be such that

$$
c_{1}^{p_{1}} \ldots c_{r}^{p_{r}}>e^{\varepsilon} .
$$

Finally, let $x \in(0, \infty)$ and choose $n_{0} \in \mathbb{N}$ such that for every $n \geq n_{0}$,

$$
(R / x)^{1 / n}<e^{\varepsilon}
$$

In view of (9) there exists a $\delta>0$ such that

$$
c_{1}^{\xi_{1}} \ldots c_{r}^{\xi_{r}}>e^{\varepsilon}
$$

for every $\xi=\left(\xi_{1}, \ldots, \xi_{r}\right) \in \mathbb{R}^{r}$ satisfying

$$
\|\xi-p\|<\delta
$$

where $p=\left(p_{1}, \ldots, p_{r}\right)$ and $\|\cdot\|$ denotes the maximum norm in $\mathbb{R}^{r}$. Now, if $n \geq n_{0}$ and $k_{1}, \ldots, k_{r} \in \mathbb{N} \cup\{0\}$ are such that

$$
\left\|\left(\frac{k_{1}}{n}-p_{1}, \ldots, \frac{k_{r}}{n}-p_{r}\right)\right\|<\delta
$$


then (see (10)-(12)) for $n \geq n_{0}$,

$$
c_{1}^{k_{1}} \ldots c_{r}^{k_{r}} x=\left(c_{1}^{k_{1} / n} \ldots c_{r}^{k_{r} / n}\right)^{n} x>e^{n \varepsilon} x>\frac{R}{x} x=R .
$$

For every $n \in \mathbb{N}$, put

$$
\begin{aligned}
K_{n} & :=\left\{\left(k_{1}, \ldots, k_{r}\right) \in \Delta_{n}: c_{1}^{k_{1}} \ldots c_{r}^{k_{r}} x \geq R\right\}, \\
L_{n} & :=\left\{\left(k_{1}, \ldots, k_{r}\right) \in \Delta_{n}:\left\|\left(k_{1} / n-p_{1}, \ldots, k_{r} / n-p_{r}\right)\right\|<\delta\right\}, \\
M_{n} & :=\Delta_{n} \backslash K_{n}, \quad P_{n}:=\Delta_{n} \backslash L_{n} .
\end{aligned}
$$

In view of (13) we have $L_{n} \subset K_{n}$ and $M_{n} \subset P_{n}$ for $n \geq n_{0}$.

Let $(\Omega, \mathcal{A}, \mathcal{P})$ be a probability space and let $\left(\mathbb{Y}_{n}\right)_{n \in \mathbb{N}}$ be a sequence of vector-valued random variables defined by

$$
\mathbb{Y}_{n}:=\frac{\mathbb{X}_{n}}{n}-p
$$

where $\mathbb{X}_{n}: \Omega \rightarrow \mathbb{R}^{r}, n \in \mathbb{N}$, are vector-valued random variables with polynomial distribution. Lemma 2.1 implies that for every $\eta>0$,

$$
\lim _{n \rightarrow \infty} \mathcal{P}\left(\left\|\mathbb{Y}_{n}\right\|<\eta\right)=1
$$

In particular, we have

$$
\sum_{\left(k_{1}, \ldots, k_{r}\right) \in L_{n}} \frac{n !}{k_{1} ! \ldots k_{r} !} p_{1}^{k_{1}} \ldots p_{r}^{k_{r}}=\mathcal{P}\left(\left\|\mathbb{Y}_{n}\right\|<\delta\right) \underset{n \rightarrow \infty}{\longrightarrow} 1 .
$$

Using (4), (7), (8), (13) and (14) we get

$$
\begin{aligned}
|g(x)|= & \mid \sum_{\left(k_{1}, \ldots, k_{r}\right) \in K_{n}} \frac{n !}{k_{1} ! \ldots k_{r} !} p_{1}^{k_{1}} \ldots p_{r}^{k_{r}} g\left(c_{1}^{k_{1}} \ldots c_{r}^{k_{r}} x\right) \\
& +\sum_{\left(k_{1}, \ldots, k_{r}\right) \in M_{n}} \frac{n !}{k_{1} ! \ldots k_{r} !} p_{1}^{k_{1}} \ldots p_{r}^{k_{r}} g\left(c_{1}^{k_{1}} \ldots c_{r}^{k_{r}} x\right) \mid \\
\geq & \sum_{\left(k_{1}, \ldots, k_{r}\right) \in K_{n}} \frac{n !}{k_{1} ! \ldots k_{r} !} p_{1}^{k_{1}} \ldots p_{r}^{k_{r}} g\left(c_{1}^{k_{1}} \ldots c_{r}^{k_{r}} x\right) \\
& -\sum_{\left(k_{1}, \ldots, k_{r}\right) \in M_{n}} \frac{n !}{k_{1} ! \ldots k_{r} !} p_{1}^{k_{1}} \ldots p_{r}^{k_{r}}\left|g\left(c_{1}^{k_{1}} \ldots c_{r}^{k_{r}} x\right)\right| \\
\geq & D \sum_{\left(k_{1}, \ldots, k_{r}\right) \in L_{n}} \frac{n !}{k_{1} ! \ldots k_{r} !} p_{1}^{k_{1}} \ldots p_{r}^{k_{r}} \\
& -B_{R} \sum_{\left(k_{1}, \ldots, k_{r}\right) \in P_{n}} \frac{n !}{k_{1} ! \ldots k_{r} !} p_{1}^{k_{1}} \ldots p_{r}^{k_{r}} \\
\geq & D \mathcal{P}\left(\left\|\mathbb{Y}_{n}\right\|<\delta\right)-B_{R} \mathcal{P}\left(\left\|\mathbb{Y}_{n}\right\| \geq \delta\right) \underset{n \rightarrow \infty}{\longrightarrow} D .
\end{aligned}
$$


Since $x>0$ was arbitrary this shows that $|g(x)| \geq D$ for $x \in(0, \infty)$. But $D>0$ was arbitrary as well, thus $|g(x)|=\infty, x \in(0, \infty)$, which contradicts the boundedness of $g$ at 0 . This contradiction shows that $a<\infty$. An analogous argument may be used to show that $a>-\infty$, too.

To prove that $g=a$ fix $\eta>0$ and let $R>0$ be such that

$$
|g(x)-a|<\eta
$$

for every $x \in[R, \infty)$. Let $x \in(0, \infty)$. From (4), (7) and (13)-(15) we get

$$
\begin{aligned}
|g(x)-a| \leq & \sum_{\left(k_{1}, \ldots, k_{r}\right) \in K_{n}} \frac{n !}{k_{1} ! \ldots k_{r} !} p_{1}^{k_{1}} \ldots p_{r}^{k_{r}}\left|g\left(c_{1}^{k_{1}} \ldots c_{r}^{k_{r}} x\right)-a\right| \\
& +\sum_{\left(k_{1}, \ldots, k_{r}\right) \in M_{n}} \frac{n !}{k_{1} ! \ldots k_{r} !} p_{1}^{k_{1}} \ldots p_{r}^{k_{r}}\left|g\left(c_{1}^{k_{1}} \ldots c_{r}^{k_{r}} x\right)-a\right| \\
\leq & \eta+\left(B_{R}+|a|\right) \sum_{\left(k_{1}, \ldots, k_{r}\right) \in P_{n}} \frac{n !}{k_{1} ! \ldots k_{r} !} p_{1}^{k_{1}} \ldots p_{r}^{k_{r}} \\
\leq & \eta+\left(B_{R}+|a|\right) \mathcal{P}\left(\left\|\mathbb{Y}_{n}\right\| \geq \delta\right) \underset{n \rightarrow \infty}{\longrightarrow} \eta
\end{aligned}
$$

Since $x \in(0, \infty)$ and $\eta>0$ were arbitrary, we get our assertion.

To prove the remaining part of the assertion it is enough to observe that if $g$ solves the equation $(\mathrm{E})$ and $\left(\mathrm{B}_{1}\right)-\left(\mathrm{B}_{3}\right)$ are satisfied then the function

$$
G(x):=g(1 / x), \quad x \in(0, \infty),
$$

satisfies (E) with $c_{i}$ replaced by $C_{i}:=1 / c_{i}, i \in\{1, \ldots, r\}$, and $G, C_{1}, \ldots, C_{r}$ satisfy $\left(\mathrm{A}_{1}\right)-\left(\mathrm{A}_{3}\right)$, hence $G(x)=a, x \in(0, \infty)$.

Let us note that the assumptions on $g$ are essential, and even high regularity of solutions does not guarantee uniqueness.

ExAmple 3.1. The function $g:(0, \infty) \rightarrow \mathbb{R}$ given by $g(x)=1 / x$ satisfies $\left(\mathrm{B}_{1}\right),\left(\mathrm{B}_{2}\right)$ and solves the equation

$$
g(x)=\frac{3}{4} g(3 x)+\frac{1}{4} g\left(\frac{x}{3}\right), \quad x \in(0, \infty) .
$$

Note that

$$
3^{3 / 4} \cdot\left(\frac{1}{3}\right)^{1 / 4}>1
$$

and thus $\left(\mathrm{B}_{3}\right)$ does not hold.

EXAMPLE 3.2. The function $g:=\mathrm{id} \mid(0, \infty)$ solves the equation

$$
g(x)=\frac{1}{2} g\left(\frac{3}{2} x\right)+\frac{1}{2} g\left(\frac{1}{2} x\right), \quad x \in(0, \infty) .
$$


and satisfies $\left(\mathrm{A}_{1}\right)$ and $\left(\mathrm{A}_{2}\right)$. However,

$$
(3 / 2)^{1 / 2} \cdot(1 / 2)^{1 / 2}<1
$$

and thus $\left(\mathrm{A}_{3}\right)$ does not hold.

The above examples also show that Proposition 3.1 does not hold when $\left(\mathrm{A}_{2}\right)$ and $\left(\mathrm{A}_{3}\right)$ are satisfied, but $\left(\mathrm{A}_{1}\right)$ is not (Example 3.1) or $\left(\mathrm{B}_{2}\right)$ and $\left(\mathrm{B}_{3}\right)$ are satisfied while $\left(\mathrm{B}_{1}\right)$ is not (Example 3.2). However, observe that in both cases nonconstant solutions are of the form $x \rightarrow x^{\lambda}$ where $\lambda$ is a nonzero solution of the respective characteristic equation $(\lambda=-1$ for the equation in Example 3.1, and $\lambda=1$ in Example 3.2). It turns out that this is not accidental. More exactly, we have the following result concerning the case where the characteristic equation (3) has a nonzero root (cf. our comment before Lemma 2.2 on the size of $\Lambda$ ).

THEOREM 3.1. Assume that $(\mathrm{H})$ holds and suppose that the set $\Lambda$ of roots of the characteristic equation (3) has two elements. Set

$$
\mu:=\min \Lambda, \quad \nu:=\max \Lambda .
$$

Let $g:(0, \infty) \rightarrow \mathbb{R}$ be a solution of equation (E) and define for every $\lambda \in \mathbb{R}$ the function $g_{\lambda}:(0, \infty) \rightarrow \mathbb{R}$ by

$$
g_{\lambda}(x)=x^{-\lambda} g(x)
$$

If

$\left(\alpha_{1}\right) \quad g_{\nu}$ is bounded in a vicinity of 0 and

$\left(\alpha_{2}\right) \quad \lim _{x \rightarrow \infty} g_{\nu}(x)=a \in[-\infty, \infty]$,

then $a \in \mathbb{R}$ and $g(x)=a x^{\nu}, x \in(0, \infty)$.

If

$$
\begin{gathered}
g_{\mu} \text { is bounded in a vicinity of } \infty \quad \text { and } \\
\lim _{x \rightarrow 0^{+}} g_{\mu}(x)=a \in[-\infty, \infty],
\end{gathered}
$$

then $a \in \mathbb{R}$ and $g(x)=a x^{\mu}, x \in(0, \infty)$.

Pro of. Suppose that $\left(\mathrm{A}_{3}\right)$ holds (cf. Proposition 3.1). According to Lemma 2.2, if $\Lambda$ consists of two elements then $c_{1}<1<c_{r}$, and hence $\mu<0=\nu$. Conditions $\left(\alpha_{1}\right)$ and $\left(\alpha_{2}\right)$ mean simply that $g$ satisfies $\left(\mathrm{A}_{1}\right)$ and $\left(\mathrm{A}_{2}\right)$ of Proposition 3.1, and the first part of the assertion follows. To prove the second part, note (cf. Introduction) that

$$
g_{\mu}(x)=\sum_{i=1}^{r} \widetilde{p}_{i} g_{\mu}\left(c_{i} x\right),
$$


where $\widetilde{p}_{i}=p_{i} c_{i}^{\mu}, i \in\{1, \ldots, r\}$. Now, from Lemma 2.2(ii) we infer that $\widetilde{p}_{i}, c_{i}$, $i \in\{1, \ldots, r\}$ and $g_{\mu}$ satisfy conditions $\left(\mathrm{B}_{1}\right)-\left(\mathrm{B}_{3}\right)$ of Proposition 3.1. Hence the second part of the assertion follows.

The proof in the case where $\left(\mathrm{B}_{3}\right)$ holds is analogous.

Remark 3.1. The condition $\left(\mathrm{A}_{3}\right)$ holds in particular if $c_{1}>1$. It turns out that in this case assumption $\left(A_{1}\right)$ is redundant. Indeed, equation $(E)$ then implies that if (8) holds for $x \geq R$ then it holds for $x \geq R / c_{1}>R$ as well. An easy induction shows that (8) has to hold for every $x>0$, which, as in the proof of Proposition 3.1, implies that $a \in \mathbb{R}$. Now, an analogous argument shows that $|g(x)-a|<\varepsilon$ for every $\varepsilon>0$ and every $x>0$. In other words, $g=a$.

Similarly, if $c_{r}<1$ then $\left(\mathrm{B}_{3}\right)$ holds and $\left(\mathrm{B}_{1}\right)$ is redundant. Thus (cf. Lemma 2.2) we can state the following.

TheOREm 3.2. Assume that $(\mathrm{H})$ holds and suppose that $\Lambda=\{0\}$. If either $c_{1}>1$ and $\left(\mathrm{A}_{2}\right)$ holds or $c_{r}<1$ and $\left(\mathrm{B}_{2}\right)$ holds then $a \in \mathbb{R}$ and $g(x)=a$, $x \in(0, \infty)$.

REMARK 3.2. Note that the second part of the above theorem was proved by J. Baker in [1] (Proposition 2), under the assumption that $a \in \mathbb{R}$.

The assumption on $g$ may also be relaxed in some other cases, not covered by our theorems. As an example, we prove a result on solutions of equation (E) with $r=2$ and $c_{2}=c_{1}^{-1}$.

Consider the equation

$$
g(x)=p g(c x)+(1-p) g\left(\frac{1}{c} x\right),
$$

where $g:(0, \infty) \rightarrow \mathbb{R}$ is the unknown function, $p \in(0,1)$ and $c \in(0, \infty) \backslash\{1\}$. First, we prove the following

LEMma 3.1. If $g$ satisfies equation (16) then for every $n \in \mathbb{N}$,

$$
g(x)=p_{n} g\left(c^{n} x\right)+\left(1-p_{n}\right) g\left(\frac{1}{c} x\right), \quad x \in(0, \infty),
$$

where

$$
p_{n}=\frac{p_{n-1} p}{1-p+p_{n-1} p}, \quad n \geq 2, \quad p_{1}=p .
$$

In particular,

$$
\lim _{n \rightarrow \infty} p_{n}=0, \quad p \in(0,1 / 2] .
$$

Proof. A simple proof of (17) and (18) will be omitted. To prove (19) define for $p \in(0,1)$ the function $f_{p}:(0,1) \rightarrow(0,1)$ by

$$
f_{p}(t):=\frac{p t}{1-p+p t}, \quad t \in(0,1) .
$$


We have

$$
f_{p}\left(p_{n}\right)=p_{n+1}=f_{p}^{n+1}(p), \quad n \in \mathbb{N} .
$$

If $p \in(0,1 / 2]$ then for every $t \in(0,1)$ we get

$$
t-f_{p}(t)=\frac{t(1-2 p+p t)}{1-p+p t}>0 .
$$

Hence $f_{p}(t)<t$ and $\lim _{n \rightarrow \infty} f_{p}^{n}(t)=0$, which ends the proof of (19).

Using Lemma 3.1 we prove

TheOREM 3.3. Let $c>1$ and let $g:(0, \infty) \rightarrow \mathbb{R}$ satisfy equation (16). If either

(i) $p \in(0,1 / 2]$ and $\lim _{x \rightarrow \infty} g(x)=A \in \mathbb{R}$, or

(ii) $p \in[1 / 2,1)$ and $\lim _{x \rightarrow 0^{+}} g(x)=A \in \mathbb{R}$,

then $g=A$.

P r o of. Suppose that (i) holds. From Lemma 2.2 we get

$$
g(x)=g\left(\frac{1}{c} x\right), \quad x \in(0, \infty),
$$

and the assumption on $c$ implies that

$$
g(x)=A, \quad x \in(0, \infty) .
$$

Assume now that (ii) holds. Setting $b:=1 / c$ and $q:=1-p$ we can write equation (16) in the form

$$
g(x)=q g(b x)+(1-q) g\left(\frac{1}{b} x\right), \quad x \in(0, \infty) .
$$

Applying Lemma 2.2 to the above equation (with $b$ instead of $c$ and $q$ instead of $p$ ) we get for every $x \in(0, \infty)$ and $n \in \mathbb{N}$,

$$
g(x)=q_{n} g\left(b^{n} x\right)+(1-q) g\left(\frac{1}{b} x\right),
$$

where

$$
q_{n}=\frac{q_{n-1} q}{1-q+q_{n-1} q}, \quad n \geq 2, \quad q_{1}=q .
$$

Since $q \in(0,1 / 2]$ we get $\lim _{n \rightarrow \infty} q_{n}=0$, moreover, $\lim _{n \rightarrow \infty} b^{n}=0$. Letting $n \rightarrow \infty$ in $(20)$ we get

$$
g(x)=g\left(\frac{1}{b} x\right), \quad x \in(0, \infty)
$$

whence $g(x)=A, x \in(0, \infty)$, follows immediately.

Acknowledgements. The second author was supported by the KBN grant No. 2 P03A 04909. 


\section{References}

[1] J. A. Baker, A functional equation from probability theory, Proc. Amer. Math. Soc. 121 (1994), 767-773.

[2] G. A. Derfel, Probabilistic method for a class of functional-differential equations, Ukrain. Mat. Zh. 41 (10) (1989), 1117-1234 (in Russian).

[3] W. Feller, An Introduction to Probability Theory and its Applications, Wiley, New York, 1961.

[4] J. Ger and M. Sablik, On Jensen equation on a graph, Zeszyty Naukowe Polit. Śląskiej Ser. Mat.-Fiz. 68 (1993), 41-52.

[5] W. Jarczyk, On an equation characterizing some probability distribution, talk at the 34th International Symposium on Functional Equations, Wisła-Jawornik, June 1996.

[6] M. Laczkovich, Non-negative measurable solutions of a difference equation, J. London Math. Soc. (2) 34 (1986), 139-147.

[7] M. Pycia, A convolution inequality, manuscript.

Institute of Mathematics

Silesian University

Bankowa 14

40036 Katowice, Poland

E-mail: mssablik@us.edu.pl

Reçu par la Rédaction le 4.3.1997

Révisé le 15.6.1997 